\title{
Optics Damage Inspection for the NIF
}

\author{
C E. Thompson \\ D E Decker \\ C F. Knopp
}

This paper was prepared for submittal to

Solid State Lasers for Application to Inertial Confinement Fusion

Monterey, $\mathrm{CA}$

June 7-10, 1998

June 1998

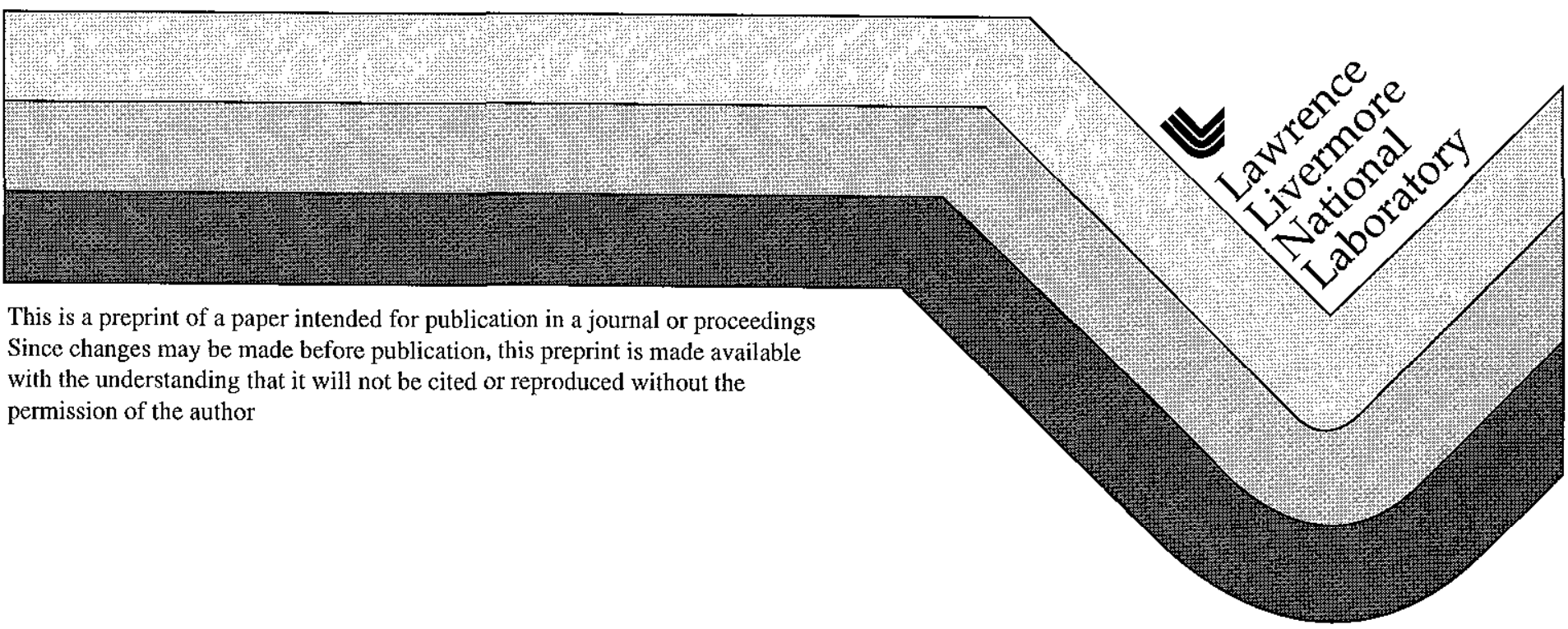




\section{DISCLAIMER}

This document was prepared as an account of work sponsored by an agency of the United States Government Neither the United States Government nor the University of California nor any of their employees, makes any warranty, express or implied, or assumes any legal liability or responsibility for the accuracy, completeness, or usefulness of any information, apparatus, product, or process disclosed, or represents that its use would not infringe privately owned rights Reference herein to any specific commercial product, process, or service by trade name, trademark, manufacturer, or otherwise, does not necessarily constitute or imply its endorsement, recommendation, or favoring by the United States Government or the University of California The views and opinions of authors expressed herein do not necessarily state or reflect those of the United States Government or the University of California, and shall not be used for advertising or product endorsement purposes 


\title{
Optics damage inspection for the NIF
}

\author{
C. E Thompson, C. F. Knopp, D. E. Decker \\ Lawrence Livermore National Laboratory \\ P. O. Box 808 \\ Livermore, CA 94550 \\ Tel. (925)422-5375 FAX: (925)422-0940 \\ E-mail: thompson4@1lnl.gov
}

\begin{abstract}
Two optics damage inspection systems will be implemented on the National Ignition Facility (NIF), one to inspect optics within the laser and transport sections and the other to inspect the final optics at the target chamber. Both systems use dark-field imaging technology to enhance resolution of defects. Details of each system design will be provided. A functional optics damage inspection system prototype, using dark-field imaging technology, is currently in operation on the Beamlet laser. This system provides us with the opportunity to measure non-ideal optical surfaces expected to be present on NIF. Prototype details and performance will be presented.
\end{abstract}

Keywords: NIF, optics damage inspection, dark-field imaging, Beamlet

\section{INTRODUCTION}

The National Ignition Facility is the latest in a series of laser facilities built by the Lawrence Livermore National Laboratory (LLNL) for the U.S. Department of Energy (DOE) to perform inertial confinement fusion (ICF) research. This laser facility, currently in the detailed design phase, consists of 192 beams housed in two laser bays. The laser bays, along with their companion switchyards, are arranged in a " $U$ " configuration around a target bay. The laser is designed to operate at fluence levels near the damage threshold of optical materials in order to achieve maximum efficiency. Each beam in the power, transport and final optics section has an area of approximately $400 \mathrm{~mm} \mathrm{x} 400 \mathrm{~mm}$. There are 38 large aperture optics in each beamline, five of which provide a vacuum to air interface.

Damage inspection of the large aperture components is required for several reasons to minimize the risk to personnel and to reduced the risk to equipment due to an implosion of vacuum loaded optics, to reduce the risk of additional component loss caused by diffraction of damage sites, to reduce the performance degradation of the laser, and to provide the ability to remove optics (and to subsequently refurbish them) before they are damaged beyond repair.

Damage flaws are typically characterized using the half-penny model from which a critical flaw size is determined. For NIF the critical flaw size radius for large aperture vacuum loaded optics is $15 \mathrm{~mm}$. This characterization provides the requirements for on-line damage inspection: flaws greater than 0.1 of the critical size in vacuum loaded optics shall be detected, inspections on vacuum loaded optics will be conducted after every shot, the time required for inspections should be compatible with the NIF 8-hour shot cycle, and inspection of other optics will become a part of weekly maintenance. Vacuum loaded optics will be removed before flaws reach 0.25 of the critical size. 


\section{SYSTEM DESCRIPTION}

Two systems are being designed to meet the NIF damage detection requirements. The laser optics damage inspection (LODI) system measures damage in the laser, transport optics and target chamber vacuum window while the final optics damage inspection (FODI) system measures damage in the final optics.Inspection proceeds one beamline at a time. Both systems use dark-field (Schlieren) imaging to enhance detection of damage; scientificgrade CCD cameras record the images.

A description of the control system and inspection scenarios is beyond the scope of this report. Inspection sequences have, however, been preliminarily defined for betweenshot and weekly activities. Between-shot inspections are expected to take approximately 2.7 hours and will first inspect the final optics using the FODI. After the laser cools, the LODI will inspect the laser spatial filtet lenses. Weekly activities, which inspect the laser, transport and final optics, is expected to take 4.3 hours. Front-end processors control the CCD cameras and deliver images to a control and image processing system consisting of two servers. Data storage requirements have been estimated at 20 Gbytes for weekly maintenance and 3.1 Gbytes for between-shot activities. Image processing is expected to proceed at the data acquisition rate which is limited by the roving mirror movement

\subsection{Laser optics damage inspection (LODI)}

Two identical LODI systems will be installed, one in each switchyard. Figure 1 shows the engineering model for switchyard \#2 A roving mirror, which can be positioned in any of the 96 beamlines within a switchyard, ditects a full aperture probe beam from the laser bay to a companion mirrot and then to a series of mirrors which transport the signal to the LODI. LODI shares this beam transport system with the Precision Diagnostic Station (PDS). A second roving mirror, used for inspection of the transport optics and by the FODI system, can be inserted to direct a probe beam from the diagnostic toward the target chamber.

Figure 2a shows schematically the laser and diagnostic configuration for measuring damage within the laser. Mirror LM1 is aligned so that only two passes through the laser are implemented and then a $1.053 \mu \mathrm{m}$ source is injected into the transport spatial filter (TSF). The cavity and transport spatial filters image relay the laser with the result that image planes from components in various parts of the beamline may overlap each other. Isolation of beamline components is accomplished by inserting pinholes in sequence in the cavity and transport spatial filters and by computationally subtracting images if required

Figure $2 \mathrm{~b}$ shows the configuration when inspection of the transport optics or the final optics assembly is required. A source, either $1.064 \mu \mathrm{m}$ or $0.355 \mu \mathrm{m}$, is provided by the alignment/LODI system depending on whether the transport mirrors or the final optics, respectively, ate inspected. Again, pre-alignment of the laser is required. A reflection from the first surface of the final focus lens is used by LODI to inspect damage of the transport mirrors The $0355 \mu \mathrm{m}$ signal is transported through the final optics and reflected into the FODI for inspection of the final optics.

When laser damage inspection is accomplished, mirror PM10 is inserted into the PDS beamline, figure 3. PM10 directs the beam through an 18.6:1 telescope and a splitter to the CCD leg A dark stop, inserted in the focal plane of a 11 relay, blocks the low spatial frequency content of the probe signal so that only the high frequency components reach the $C C D$ These components contain the damage information. A video CCD is used 
to control alignment onto the dark stop and a mirror is inserted to reflect the probe signal into a power detector so that intensity of the probe beam can be measured. The CCD z-axis position is adjusted to image the optic under inspection and the spatial filter pinholes are inserted as described above to isolate the optic. This sequence is repeated for each beamline. The systems in each switchyard are capable of independent operation so that simultaneous data acquisition and a subsequent reduction of total inspection time may be achieved. requirements:

Table 1 summarizes the LODI key design parameters which meet the design Table 1

\begin{tabular}{|l|c|}
\hline \multicolumn{1}{|c|}{ Object location (near field) } & Laser or transport section \\
\hline Object space parameters & $372 \times 372$ \\
\hline Object size [mm x mm] & 0.00143 \\
\hline Numerical aperture & 900 \\
\hline Airy diameter $[\mu \mathrm{m}]$ & \pm 260 \\
\hline Raleigh depth of focus [mm] & 780 to 1040 \\
\hline Estimated axial depth discrimination [mm] & +110 to -30 \\
\hline Acquisition range (measured from PL2) [m] & $20 \times 20$ \\
\hline Image space parameters & 0.0265 \\
\hline Image size [mm x mm] & 49 \\
\hline Numerical aperture & \pm 0.75 \\
\hline Airy diameter [ $\mu \mathrm{m}]$ & 2.25 to 3.0 \\
\hline Raleigh depth of focus [mm] & $\sim 0.9$ \\
\hline Estimated axial depth discrimination [mm] & 0.054 \\
\hline Optical resolution in main beam [mm] & $>0.5$ \\
\hline Magnification & $<0.2$ \\
\hline Strehl ratio & \\
\hline Distortion $(\%)$ & \\
\hline
\end{tabular}

The alignment laser shown in figure 4 is used when transport optics or the final optics are inspected. The laser is a commercial YAG laser with harmonic conversion; all three wavelengths are generated simultaneously. Binary attenuators are provided to control intensity. A 10:1 reflective telescope provides the first stage of up-collimation Because of the long optical paths, up to 110 meters in some cases, beam apodization is required which is accomplished with a serrated aperture and pinhole combination to remove the diffraction effects caused by a hard aperture clip. After the spatial filter/pinhole but before the splitter, a lens inserted so that the $0.355 \mu \mathrm{m}$ signal will be collimated after exiting the $18.6: 1$ telescope.

The $1.064 \mu \mathrm{m}$ signal reflected from the final focus lens is used to inspect the transport mirrors and is introduced to the CCD in the same manner as the laser probe signal This system is also capable of viewing the target chamber vacuum window but because of the finite depth of focus cannot distinguish between the window and the doubler, tripler or the first surface of the final focus lens

\subsection{Final optics damage inspection (FODI)}


Laser beams used for inditect drive ICF experiments enter the target chambet in two concentric cones having half-angles of approximately $27^{\circ}$ and $53^{\circ}$. Direct drive experiments require an additional cone at $75^{\circ}$ Integrated optics modules (IOM) on each beamline, mounted at the target chamber wall, contain the final optics which are arranged in three groups: group 1, the vacuum window, group 2, the doubler, tripler, and lens, and group 3, two diffractive optic plates plus a debris shield. Damage on these optics is viewed, one beam at a time, with the final optics damage inspection system which is mounted in a diagnostic instrument manipulator (DIM) inside the target chamber.

Figure 4 depicts schematically the FODI and its main features. A mirror, located at target chamber center, is rotated in phi while the DIM is rotated in theta to access any indirect drive IOM. Internal to the DIM, the FODI is rotated so that the skew of the image on the CCD introduced by the phi-theta rotations is removed. Direct drive beams approximately $150^{\circ}$ from the DIM cannot be viewed by the FODI due to the grazing incidence on the mirror at chamber centet A second target chamber port, $97^{\circ}$ from the primary port, is available for mounting a second FODI for inspecting these IOMs Source illumination for the final optics is provided by the LODI alignment laser $(355 \mathrm{~nm})$ located in each switchyard

Within the FODI a telescope images the final optics onto the $2 \mathrm{k} \times 2 \mathrm{k}$ CCD camera via a dark field stop which blocks the low spatial frequency light Additional alignment lenses are provided to aid in alignment of the dark stop. The camera is moved along the $\mathrm{z}$ axis to properly image the three groups of optics. All movements are performed under computer contiol Figure 5 shows the opto-mechanical configuration. The design provides sufficient depth of field to distinguish between each IOM optical group but not to distinguish optics within a group.

Table 2 summarizes the FODI design for optics within two different groups

Table 2

\begin{tabular}{|l|c|c|}
\hline Object location (near field) & $\begin{array}{c}\text { Target chamber } \\
\text { vacuum window }\end{array}$ & Debris shield \\
\hline Object space parameters & & \\
\hline Object size [mm x mm] & $424 \times 424$ & $424 \times 424$ \\
\hline Numerical aperture & 0.002 & 0.002 \\
\hline Airy diameter $[\mu \mathrm{m}]$ & 217 & 217 \\
\hline $80 \%$ contrast depth of field $[\mathrm{mm}]$ & 46 & 46 \\
\hline $10 \%$ contrast depth of field $[\mathrm{mm}]$ & 131 & 131 \\
\hline Telecentric stop & Yes & No \\
\hline Image space parameters & & \\
\hline Image size [mm x mm] & $163 \times 163$ & $172 \times 17.2$ \\
\hline Numerical aperture & 0052 & 0.049 \\
\hline Airy diameter $[\mu \mathrm{m}]$ & 83 & 8.8 \\
\hline Pixel size $[\mu \mathrm{m}]$ & $9 \times 9$ & $9 \times 9$ \\
\hline Resolution in main beam $[\mathrm{mm}]$ & $\sim 05$ & $\sim 05$ \\
\hline Magnification & 0039 & 0041 \\
\hline Strehl ratio & 05 & 0.5 \\
\hline Distortion $(\%)$ & $<1$ & $<1$ \\
\hline
\end{tabular}




\subsection{Schlieren on-line inspection of damage (SOLID)}

The Schlieren on-line inspection of damage (SOLID) system, installed on the Beamlet laser, is a functional prototype of the NIF damage systems. Besides providing an opportunity test NIF damage inspection concepts, SOLID's objectives are to detect and monitor flaws on the final focus lens during experimental campaigns, optimize image processing procedures, and investigate how imaging real flaws affect detection. Additional objectives are to measure the effect of noise contributed by real optics and to quantify the effectiveness of isolating optics within the laser using spatial filter pinholes.

The SOLID system is located at the prime focus of the Beamlet focal plane diagnostic (FPD), figure 6. The FPD measures the performance of the final optics at a plane equivalent to target chamber center. In this configuration SOLID simulates both the LODI and FODI systems. A pickoff mirror inserted into the main beamline directs an alignment beam to a dark-field block positioned at the focal plane of the final focus lens. Following the block a lens recollimates the beam before it is introduced onto a $2 \mathrm{k} \mathrm{x} 2 \mathrm{k}$ $\mathrm{CCD}$. Various image planes are acquired by adjusting the $z$-axis position of the camera and sufficient $z$-axis motion is available to allow acquisition of all components in the main laser. Spatial resolution in the main beam has been measure at $0.6 \mathrm{~mm}$

Performance of the SOLID system is being evaluated by correlating images taken on-line with damage detected by traditional methods An early data set is shown in figure 7 where new defects observed by a manual inspection of the final optics cell were also observed by the SOLID system.

In another experiment, figure 8 , damage sites in a SOLID image taken with the transport spatial filter pinhole out are no longer evident when the pinhole is in place. This demonstrates that these damage sites are located in a component on the laser side and not on the SOLID side of the pinhole and that high spatial frequencies generated prior to the pinhole can be removed. Components in different parts of the laser can thus be isolated to identify which one has damage. This is necessary because of the imaging relaying employed in ICF lasers,

Damage detection and system testing on an operating laser is difficult because great effort is taken to maintain the optical system at a high level and consequently few, if any, damage sites are available for analysis. In order test the SOLID system with a range of damage sizes, a 12" diameter debris shield from the Shiva laser was inserted into the Beamlet beamline between the cavity spatial filter and the amplifier section. Images taken by the SOLID system of this test optic were compared with off-line images of the same optic taken by a high resolution damage mapping system and with a high resolution microscope. Figure 9 shows a small area analyzed in detail by all three systems. Damage sites in the $0.6 \mathrm{~mm}$ to approximately $2 \mathrm{~mm}$ range are easily detected by SOLID. The center of the large site, approximately $4 \mathrm{~mm}$ diameter, is not detected by SOLID which is attributed to the large dark field stop ( $350 \mu \mathrm{r})$ used in this experiment. This large stop blocks most of the low spatial frequency components that are needed to clearly fill in the center. The choice of a smaller block will solve this problem and still allow retention of the higher frequencies necessary to observe the smaller sites Data from these experiments will be used to develop automatic analysis algorithms for the NIF.

\section{SUMMARY}

Two damage inspection systems have been designed for the National Ignition Facility, one to detect damage within the laser and transport optics and another to detect 
damage within the final optics at the target chamber. A NIF functional prototype has been implemented on the Beamlet laser and data is being taken. Results from the Beamlet testing verifies that our basic approach should work for NIF. Algorithms for analyzing images are being designed using the Beamlet data.

\section{ACKNOWLEDGEMENTS}

This work was performed under the auspices of the U.S. Department of Energy by Lawrence Livermore National Laboratory under contract No. W-7405-Eng-48. 


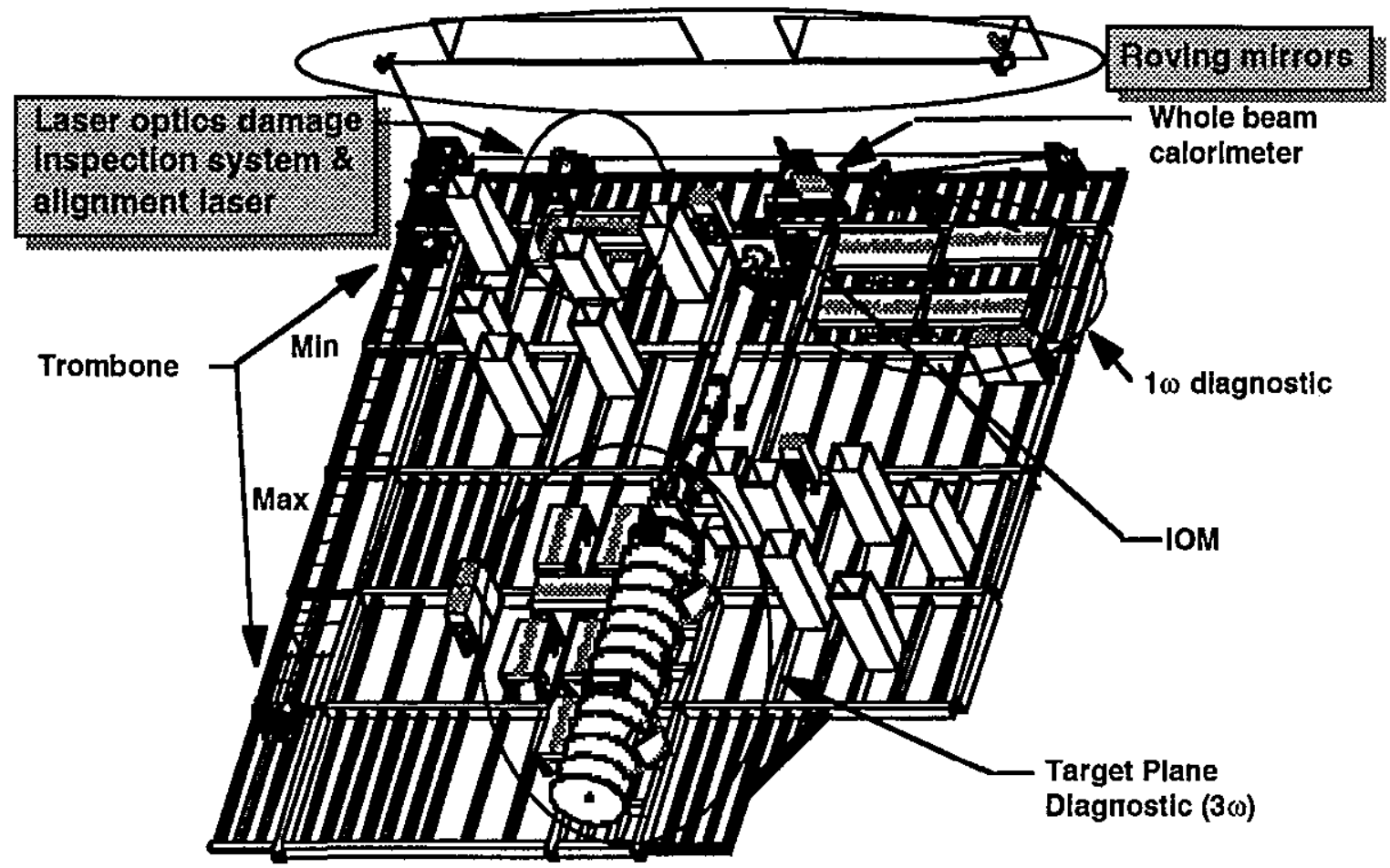

Figure 1 . The engineering model of a portion of switchyard \#2. A roving mirror selects one beam a time that is transported to the laser optics damage inspection system for data acquisition. 


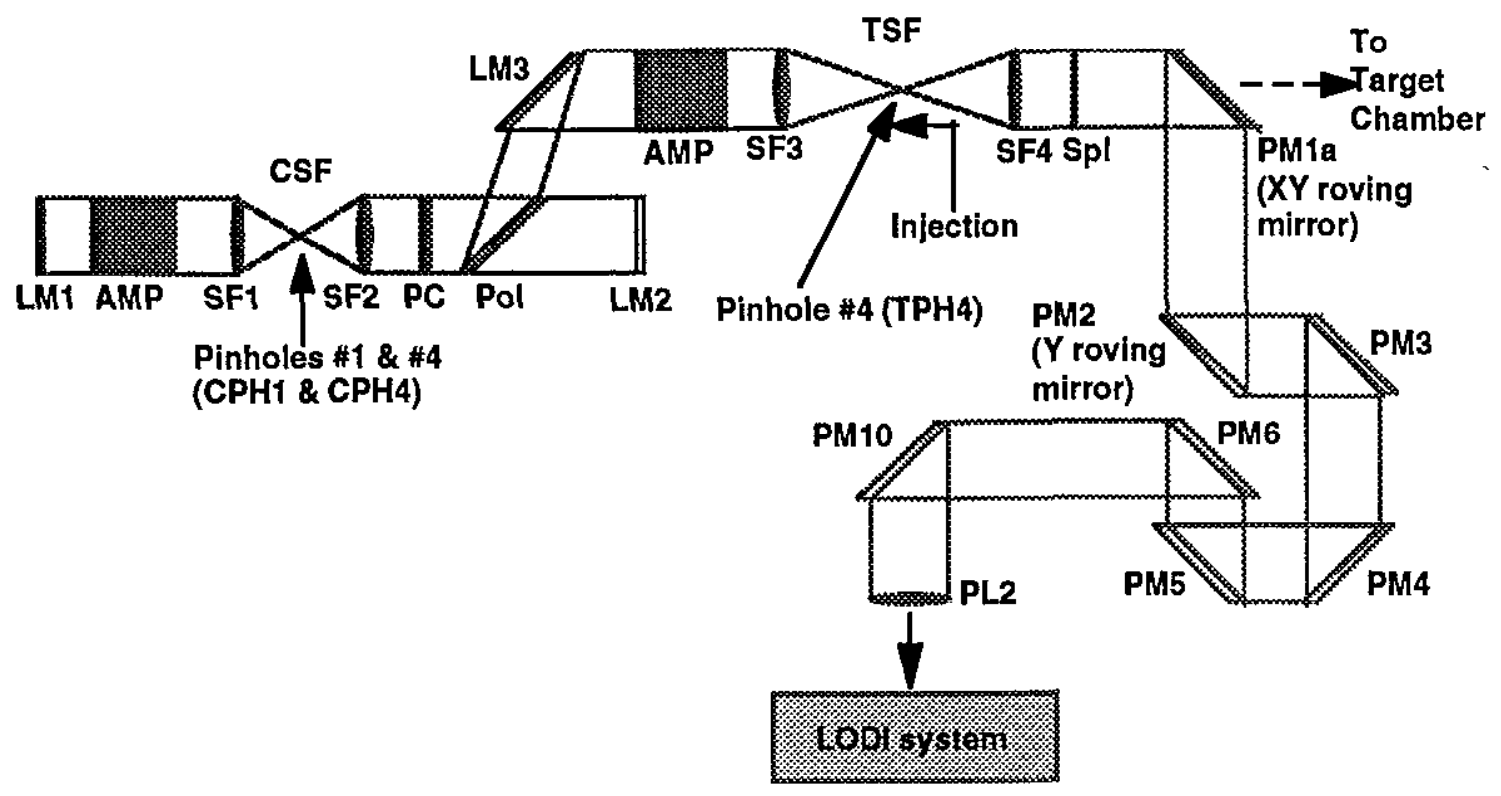

(a)

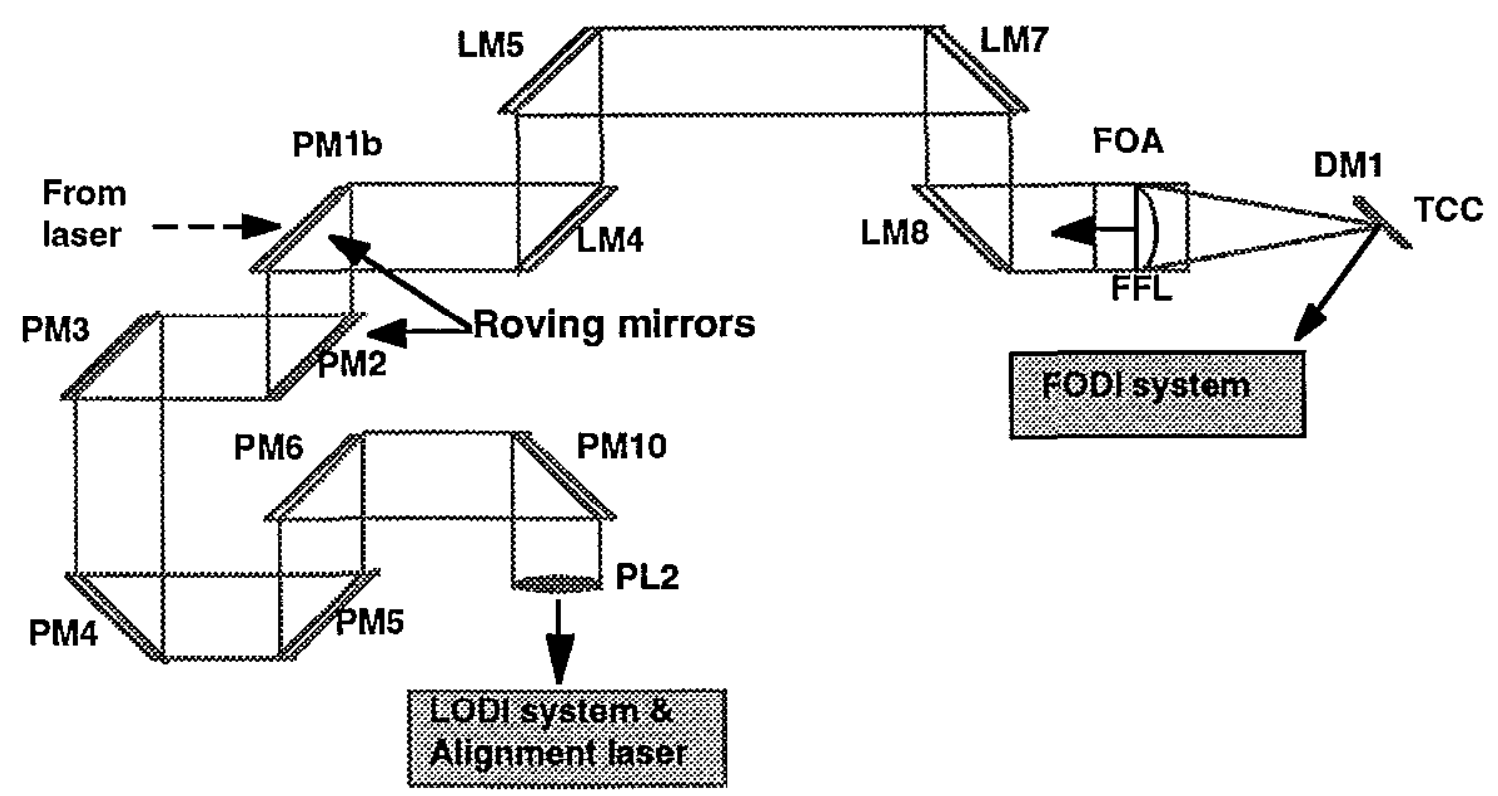

(b)

Figure 2 Schematics showing configurations for damage inspection (a) For measuring damage within the laser. (b) For measuring damage within the transport section or the final optics assembly (FOA). 


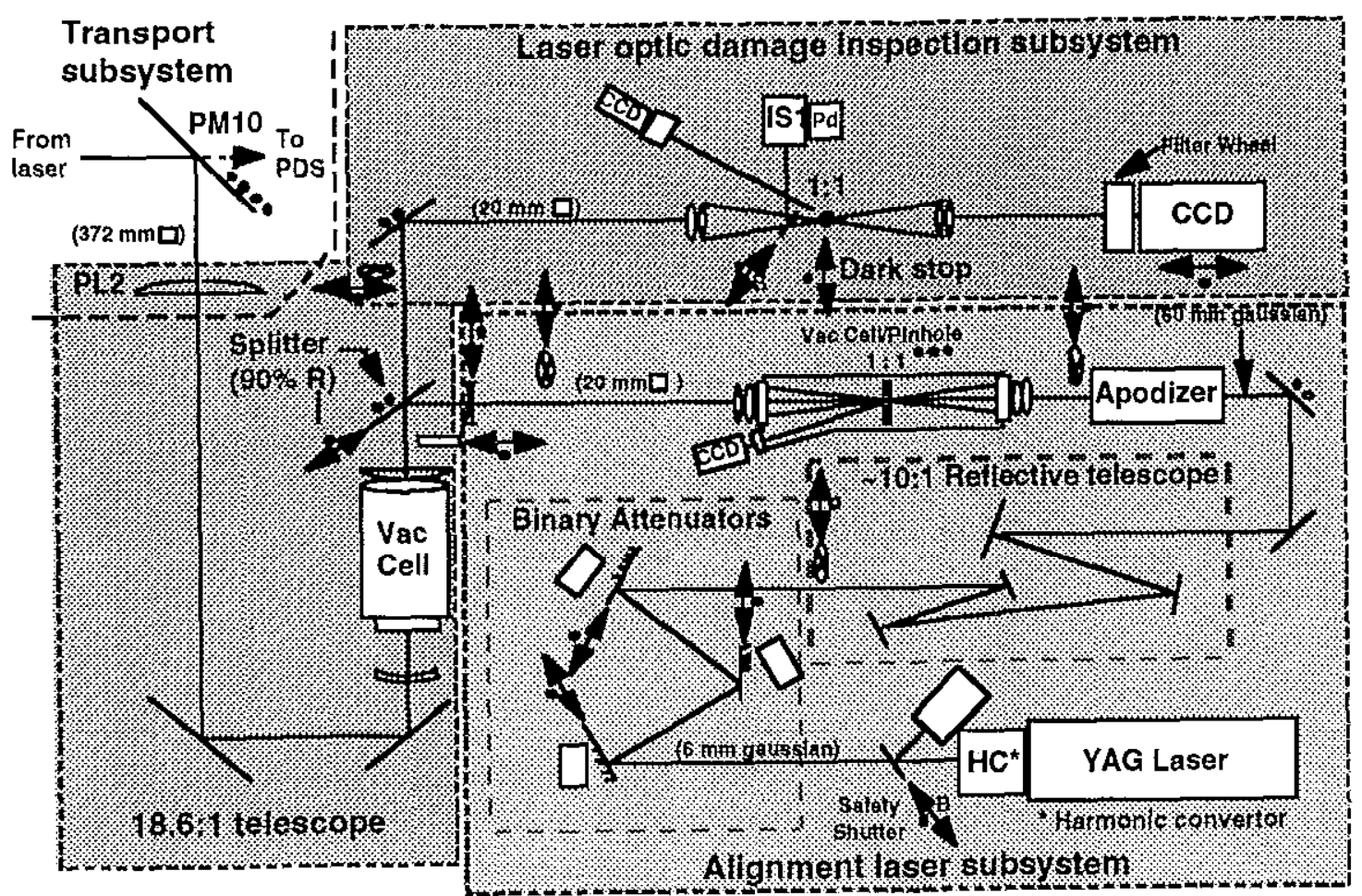

Figure 3. Schematic of the LODI and alignment laser systems The Precision Diagnostic alignment laser is the illumination source used when inspecting the transport optics and the final optics (by FODI). 


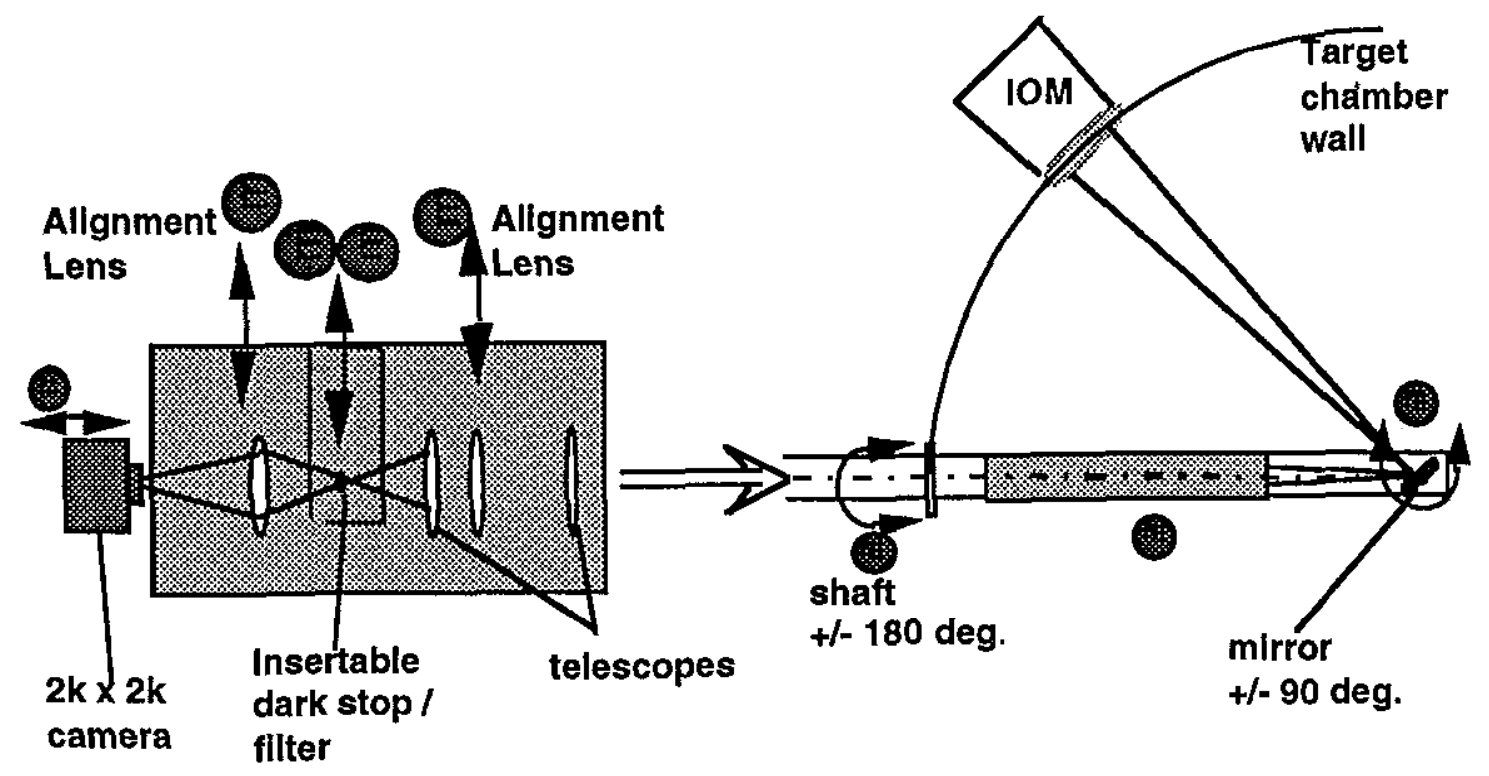

Figure 4. Schematic of the final optics damage inspection (FODI) system. Illumination at $355 \mathrm{~nm}$ is provided by the alignment lasers located in each the switchyard. 


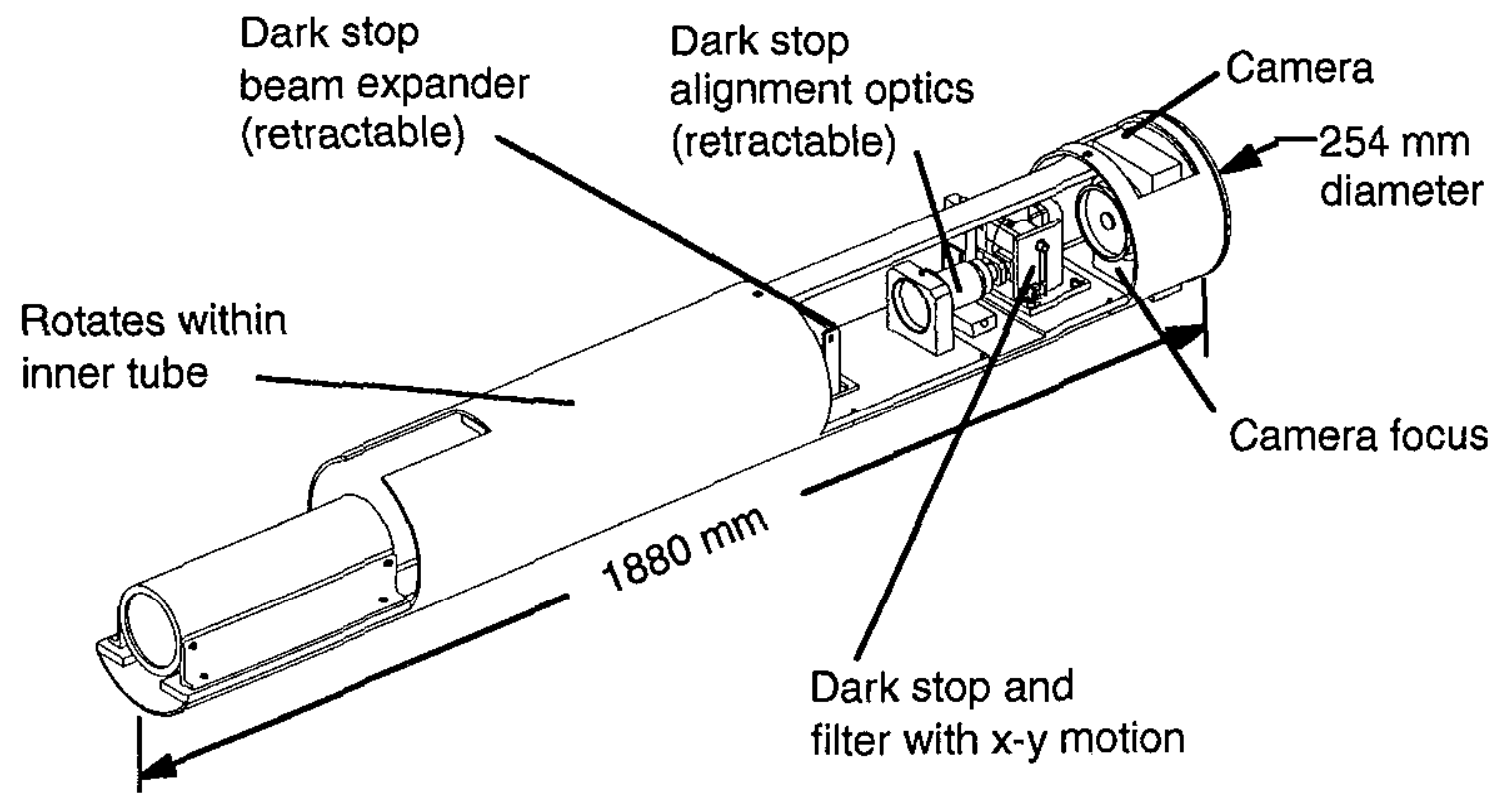

Figure 5. Opto-mechanical configuration of the FODI. 


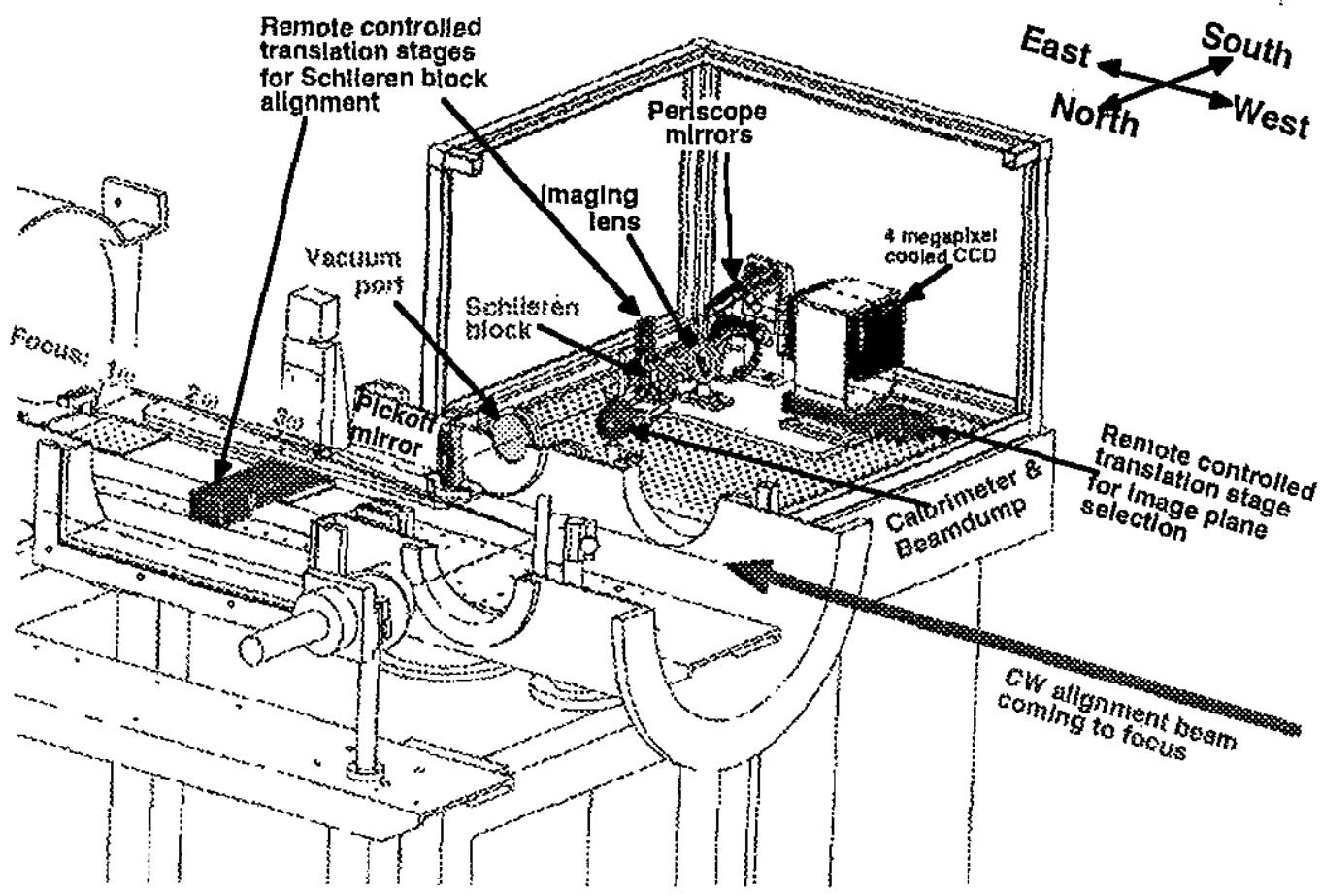

Figure 6. Engineering model of the Schlieren on-line inspection of damage (SOLID) system implemented on the Beamlet laser. 


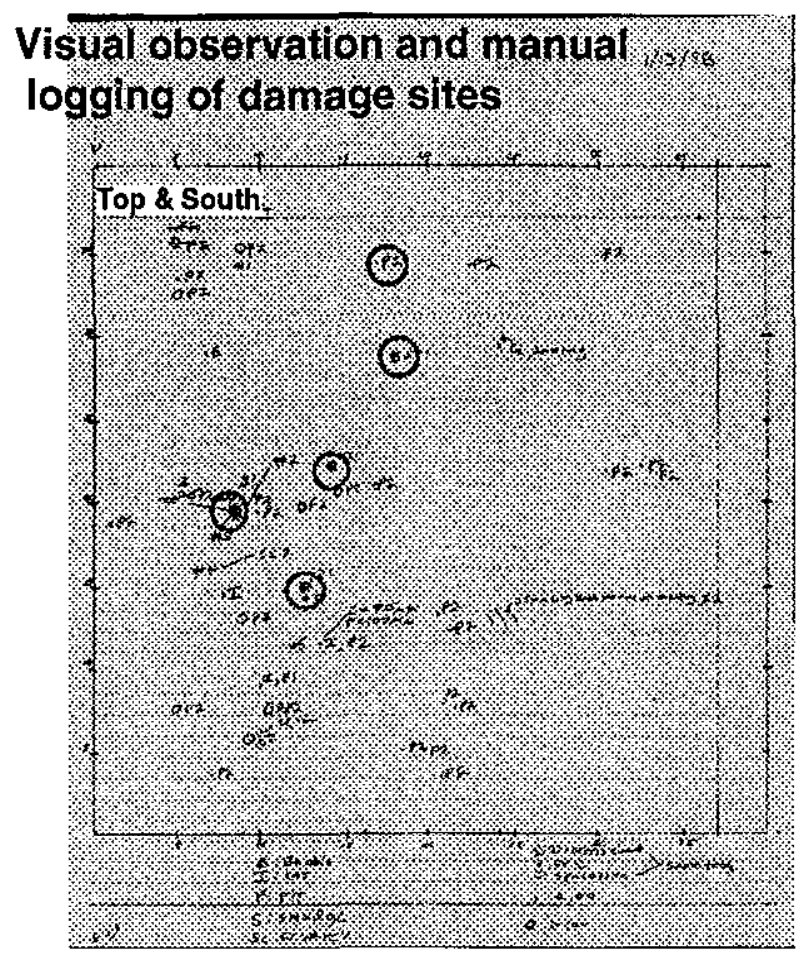

\section{SOLID image of same area}

Top \& South

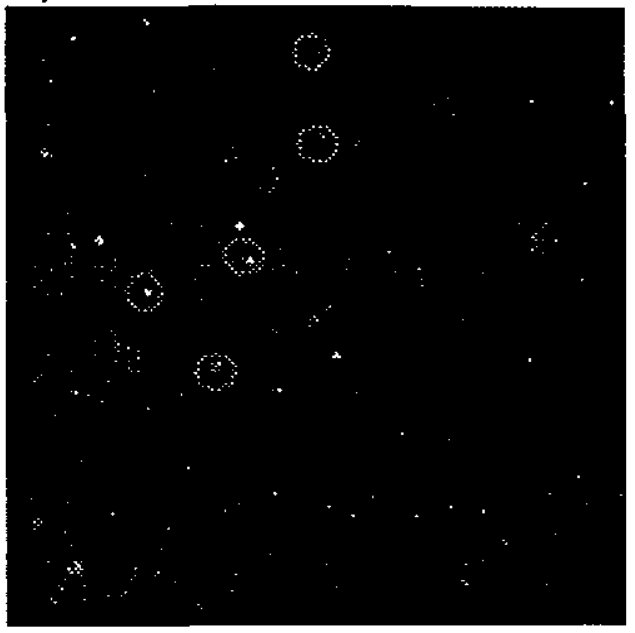

B8011307.SLD

Figure 7 . SOLID image shows new damage verified by manual inspection. 


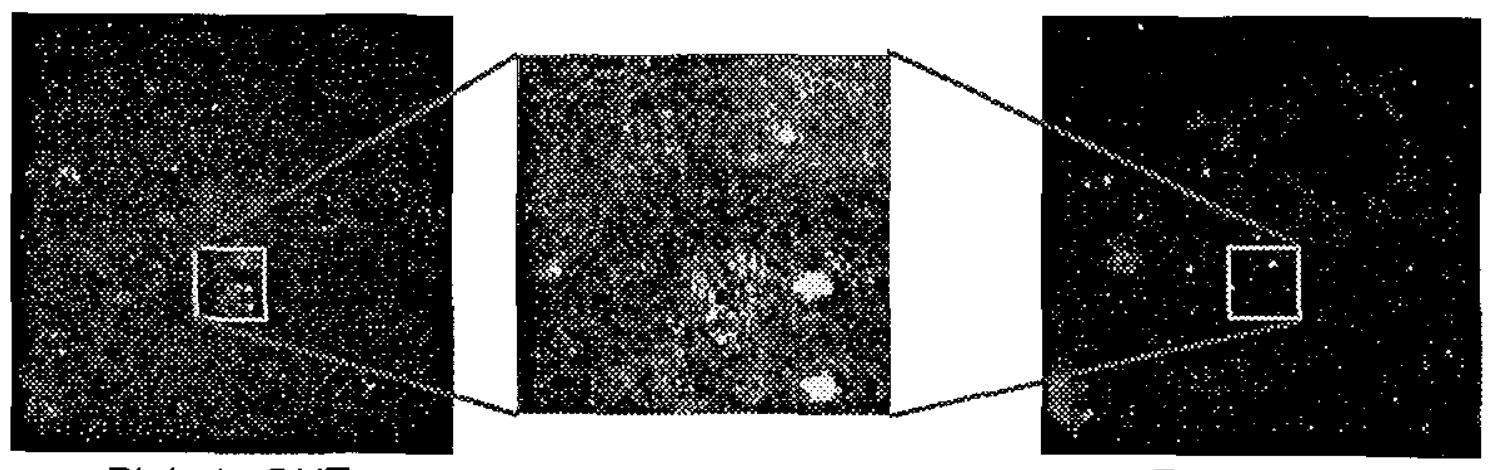

Pinhole OUT

Pinhole IN

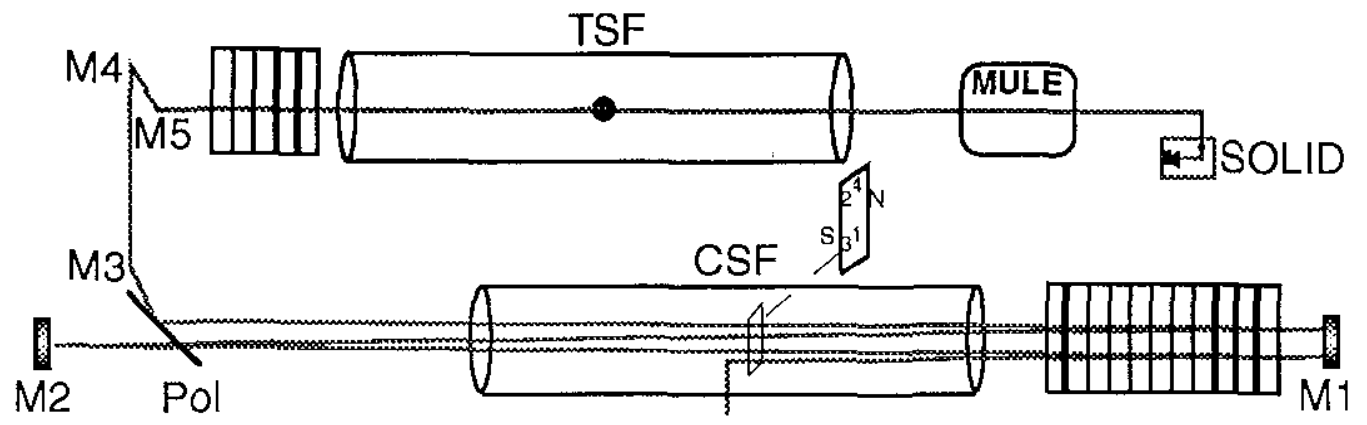

Figute 8 Insertion of the transport spatial filter (TSF) pinhole removes damage information from the laser in the SOLID image Bright spots indicating damage in lower right quadrant of center image is visible in left image but not in right 


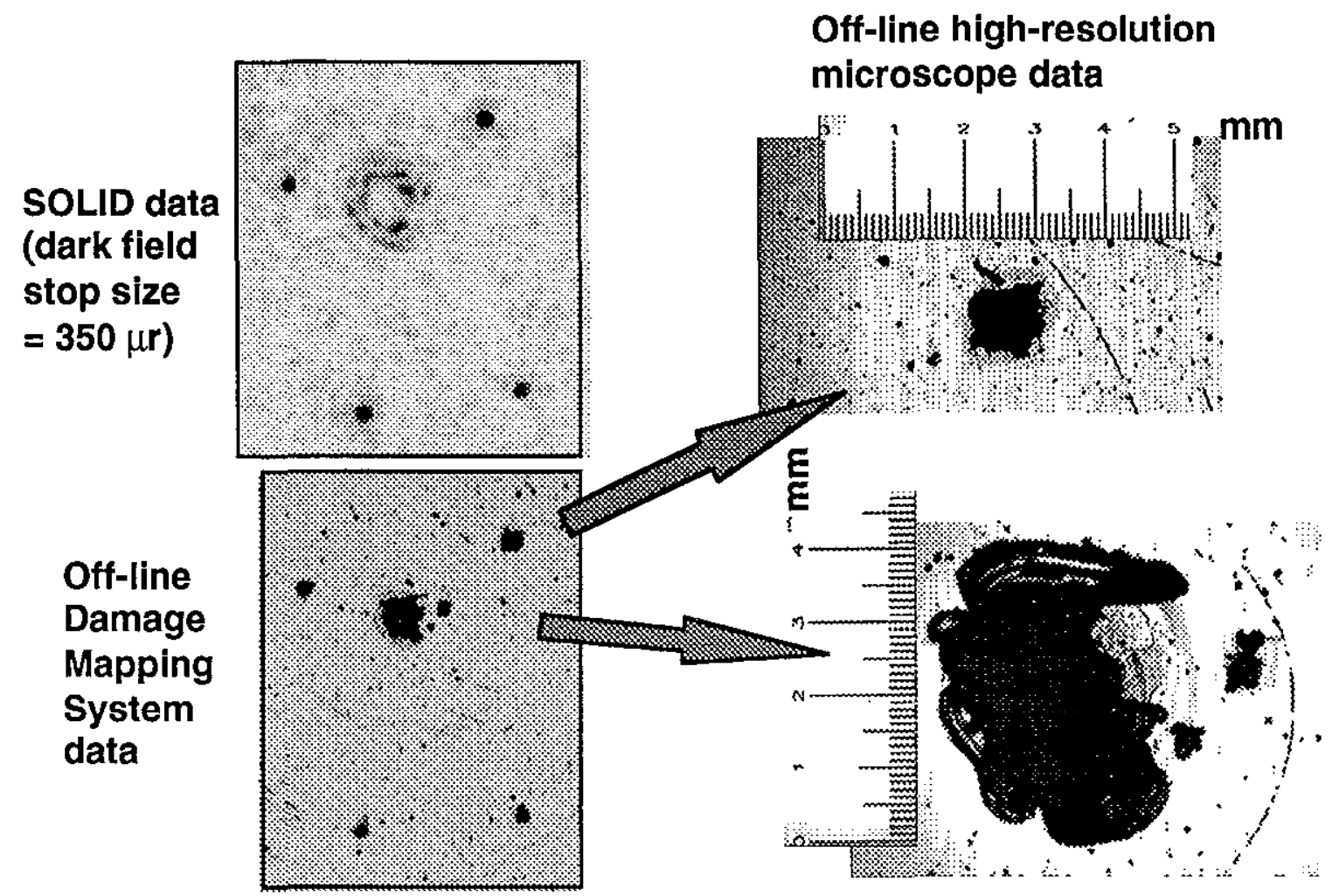

Figure 9. SOLID data from a test optic inserted in the beamline correlates well with offline, high resolution damage inspection system data The lat ge dark field stop blocks low spatial frequencies that ate tequired to fill in the center of the large defect. 


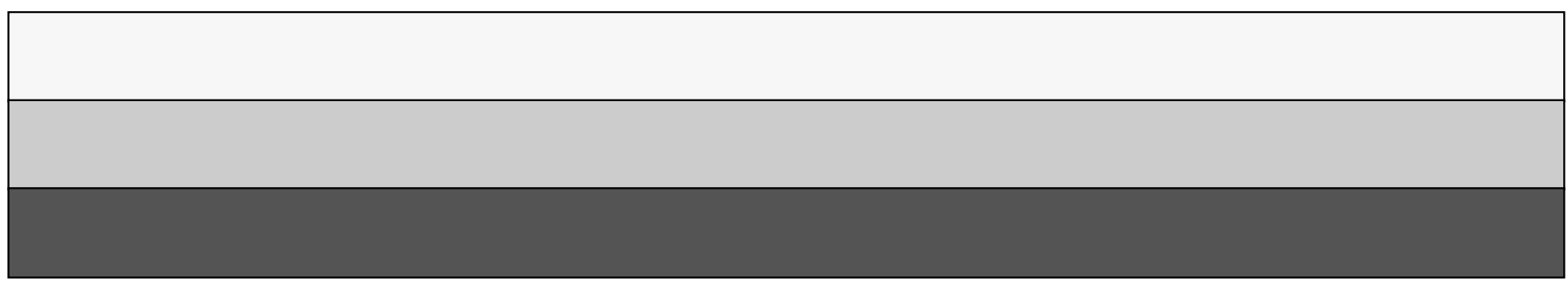

